Marquette University

e-Publications@Marquette

Mathematics, Statistics and Computer Science

Mathematics, Statistics and Computer Science,

Faculty Research and Publications

Department of

$1-1-2016$

\title{
A Hybrid Segmentation and D-Bar Method for Electrical Impedance Tomography
}

Sarah J. Hamilton

Marquette University, sarah.hamilton@marquette.edu

J.M. Reyes

Cardiff University

Samuli Siltanen

University of Helsinki

X.Zhang

Shanghai Jiaotong University

Published version. SIAM Journal on Imaging Sciences, Vol. 9, No. 2 (2016): 770-793. DOI. (C) 2016

Society for Industrial and Applied Mathematics. Used with permission. 


\title{
A Hybrid Segmentation and D-Bar Method for Electrical Impedance Tomography*
}

\author{
S. J. Hamilton ${ }^{\dagger}$, J. M. Reyes ${ }^{\ddagger}$, S. Siltanen ${ }^{\S}$, and X. Zhang $₫$
}

Abstract. The regularized D-bar method for electrical impedance tomography (EIT) provides a rigorous mathematical approach for solving the full nonlinear inverse problem directly, i.e., without iterations. It is based on a low-pass filtering in the (nonlinear) frequency domain. However, the resulting D-bar reconstructions are inherently smoothed, leading to a loss of edge distinction. In this paper, a novel method that combines a D-bar approach with the edge-preserving nature of total variation (TV) regularization is presented. The method also includes a data-driven contrast adjustment technique guided by the key functions ( $C G O$ solutions) of the D-bar method. The new $T V$-enhanced D-bar method produces reconstructions with sharper edges and improved contrast. This is achieved by using the TV-induced edges to increase the truncation radius of the scattering data in the nonlinear frequency domain, thereby increasing the radius of the low-pass filter. The algorithm is tested on numerically simulated noisy EIT data and demonstrates significant improvements in edge preservation and contrast which can be highly valuable for absolute EIT imaging.

Key words. electrical impedance tomography, D-bar method, edge preserving, scattering transform, Beltrami equation

AMS subject classification. 35R30

DOI. $10.1137 / 15 \mathrm{M} 1025992$

1. Introduction. In electrical impedance tomography (EIT) a conductive body is probed with harmless electrical currents fed into the body through electrodes at the surface, and the resulting voltages are measured at the electrodes. The goal is to recover the electrical conductivity distribution inside the body from these surface electrical measurements. EIT is useful in medical imaging, as different tissues have different conductivities, and it allows harmless and painless monitoring of patients even over long periods of time. Another application area

\footnotetext{
${ }^{*}$ Received by the editors June 17, 2015; accepted for publication (in revised form) February 12, 2016; published electronically June 1, 2016.

http://www.siam.org/journals/siims/9-2/M102599.html

${ }^{\dagger}$ Department of Mathematics, Statistics, and Computer Science, Marquette University, Milwaukee, WI 53201 (sarah.hamilton@marquette.edu). This author's research was supported by the Academy of Finland (Finnish Centre of Excellence in Inverse Problems Research 20122017, decision 250215), the SalWe Research Program for Mind and Body (Tekes - the Finnish Funding Agency for Technology and Innovation grant 1104/10), and the 2015 Summer Faculty Fellowship from Marquette University.

${ }^{\ddagger}$ School of Computer Science \& Informatics, Cardiff University, Cardiff CF24 3AA, UK (reyes.juanmanuel@gmail. com). This author's research was supported by the Academy of Finland (Finnish Centre of Excellence in Inverse Problems Research 20122017, decision 250215) and the Engineering and Physical Sciences Research Council (EPSRC), reference $\mathrm{EP} / \mathrm{K} 024078 / 1$.

${ }^{\S}$ Department of Mathematics \& Statistics, University of Helsinki, Helsinki 00014, Finland (Samuli.Siltanen@ helsinki.fi). This author's research was supported by the Academy of Finland (Finnish Centre of Excellence in Inverse Problems Research 20122017, decision 250215).

"School of Mathematical Sciences, MOE-LSC, and Institute of Natural Sciences, Shanghai Jiao Tong University, Shanghai 200240, China (xqzhang@sjtu.edu.cn). This author's research was supported by the NSFC (11101277 and 91330102), a Sino-German Center grant (GZ1025), and the 973 Program (2015CB856004).
} 


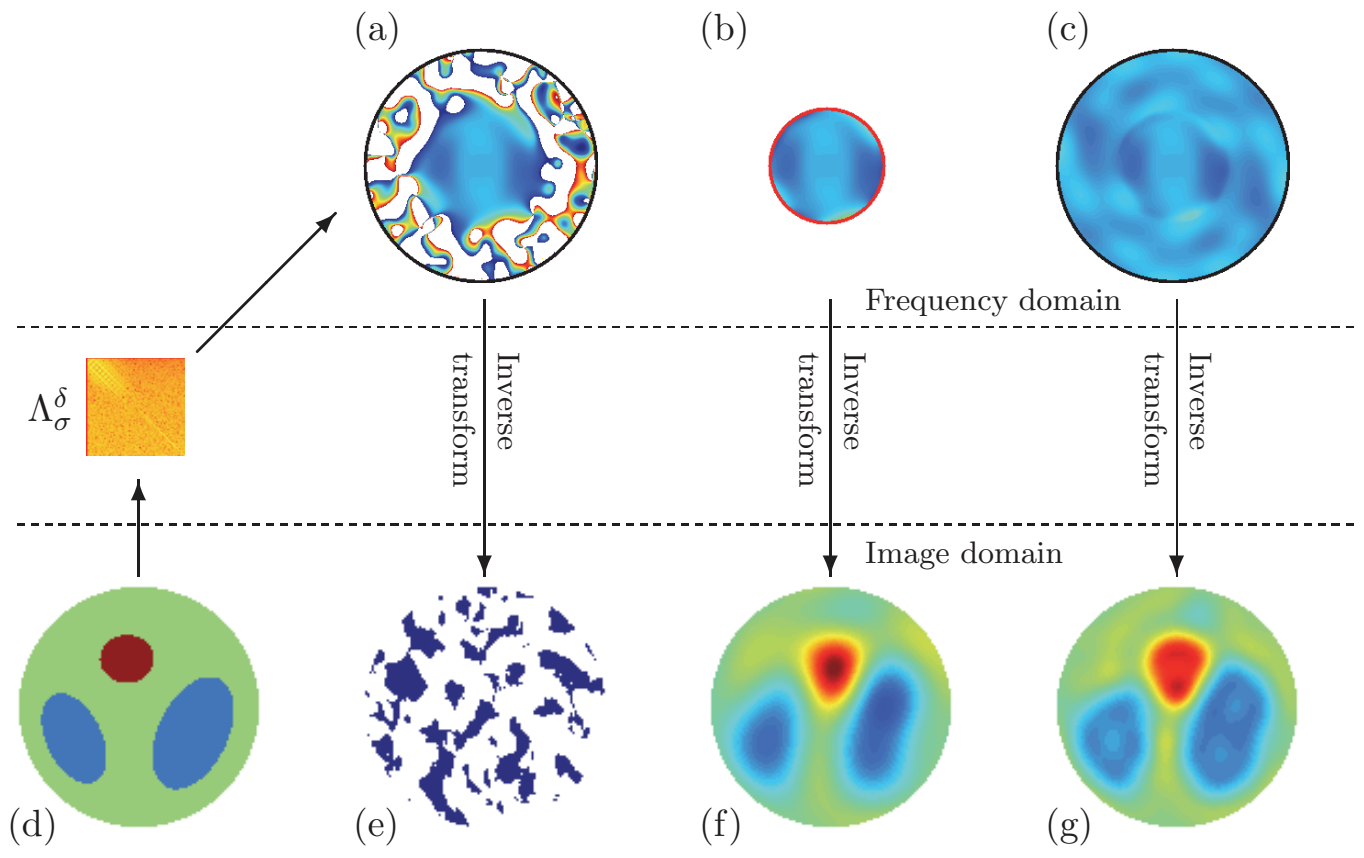

Figure 1. Schematic illustration of the nonlinear low-pass filtering approach to regularized EIT. The simulated heart-and-lungs phantom (d) gives rise to a finite voltage-to-current matrix $\Lambda_{\sigma}^{\delta}$ (orange square), which can be used to approximately determine the nonlinear Fourier transform (a). Measurement noise causes numerical instabilities in the transform (irregular white patches in (a)), leading to an unstable and inaccurate reconstruction (e). However, multiplying the transform by the characteristic function of the disc $|k|<5$ yields a low-pass-filtered transform (b), which in turn gives a noise-robust approximate reconstruction (f). The hybrid method presented in this paper uses a priori information about the conductivity to estimate the missing part of the nonlinear Fourier transform, resulting in (c). An improved reconstruction (g) is achieved.

of EIT is nondestructive testing. See $[18,41]$ for reviews of EIT and its uses.

The image reconstruction task of EIT is a nonlinear and severely ill-posed inverse problem. Therefore, EIT algorithms need to be regularized to overcome the extreme sensitivity to modeling errors and measurement noise. Among EIT algorithms, the so-called D-bar method stands out due to its unique capability of dividing the measurement information neatly into stable and unstable parts in a (nonlinear) frequency domain. See Figure 1.

Generally speaking, regularization involves complementing insufficient measurement information by a priori knowledge about the conductivity. The D-bar method does this very explicitly, assuming that the conductivity is twice continuously differentiable, which allows replacing the values of the nonlinear Fourier transform by zero in the unstable part of the frequency domain. Therefore, this low-pass filtering has the side effect that the resulting D-bar reconstructions are always smooth, as seen in the middle image of Figure 2.

In many applications of EIT, including medical imaging, it is important to see boundaries between regions of different conductivities. The standard D-bar method practice of inserting zeroes for high frequencies is not ideal, as crisp boundaries between different conductivity regions necessarily contain high frequencies.

We introduce a novel edge-enhancing method for EIT, built upon the assumption that 
Ground truth

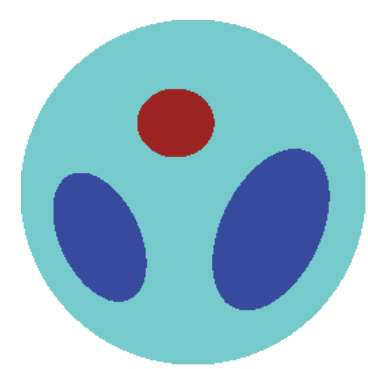

D-bar method

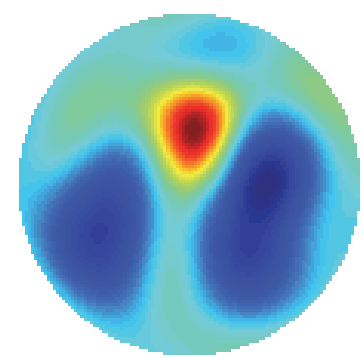

Proposed method

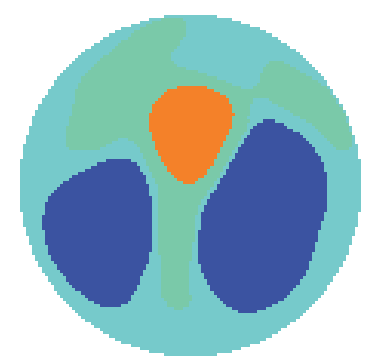

Figure 2. Left: simulated "heart-and-lungs" phantom conductivity. Middle: D-bar reconstruction based on nonlinear low-pass filtering with $0.75 \%$ relative noise added to EIT voltage data (see Figure 1). Right: reconstruction with the proposed hybrid method from the same noisy EIT data.

we know a priori that the conductivity is piecewise constant. It builds upon the stable Dbar reconstruction and increases the radius of reliable scattering data to pick up the missing high-frequency features (sharp edges and jumps) in the recovered conductivity. The method applies total variation (TV) segmentation and data-driven contrast enhancement to the Dbar reconstruction regularized by low-pass filtering with cutoff frequency $R$. We exploit the methodology in $[4,2,1]$ that allows the computation of a nonlinear Fourier transform of the discontinuous segmented image in the annulus $R-1<|k|<\tilde{R}$ for certain $\tilde{R}>R$. This new transform is added on the annulus $R<|k|<\tilde{R}$ to the original transform restricted to the disc $|k|<R-1$, and we continuously blend both transforms on the annulus $R-1<|k|<R$. From this combined scattering data on the disc $|k|<\widetilde{R}$, a new sharper D-bar conductivity reconstruction is obtained. This procedure is iterated as outlined in Figure 3.

Figure 2 shows a reconstruction from simulated EIT data with $0.75 \%$ relative noise added to the voltage data. Our nonlinear method delivers a piecewise constant and edge-preserving reconstruction. The edges are more correctly located near the boundary. This is in accordance with the basic intuition about EIT: the deeper in you try to see, the harder it gets.

Let us comment on the variety of D-bar methods we use. The three options are Schrödingertype, $2 \times 2$ (first-order) system-type, and Beltrami-type. They all have two steps: recover frequency-domain information and reconstruct via an inverse transform. Theoretically, only the Beltrami approach can deal with discontinuous conductivities. However, its second step is nonuniform in quality; see [1, section 6.3] and [5, Figure 12]. Below, we use the shortcut method introduced in [5], combining the Beltrami-type first step and the Schrödinger-type second step.

Several edge-preserving regularization methods have been suggested for EIT in the literature, including Mumford-Shah approaches [42], TV regularization, and sparsity-promoting techniques $[21,34,47,16,19,53,54,33,55]$. The method proposed in this paper is very different from all of these approaches. In the first place, it uses a D-bar image as its starting point and further uses the inverse scattering of the D-bar methodology to guide the image segmentation. Second, for the Mumford-Shah image segmentation model, we use a convex relaxation model based on TV regularization instead of an implicit level set function evolution, 
(a)

Set $j=1$ and compute the nonlinear Fourier transform $\mathbf{t}^{0}(k)$ in the stable disc $|k|<R$ from the noisy EIT data matrix $\Lambda_{\sigma}^{\delta}$.

(b)

$$
\mathbf{t}^{0}
$$

Compute the D-bar reconstruction $\sigma_{\mathrm{DB}}^{(j)}$ from the truncated transform $\mathbf{t}^{j-1}(k)$. If $j=1$, use cutoff disc $|k|<R$; else use larger disc $|k|<\widetilde{R}$.

$$
\mathbf{t}^{j-1}
$$

(c)

Use TV segmentation flow to introduce edges to the smooth image $\sigma_{\mathrm{DB}}^{(j)}$. The result $\sigma_{\mathrm{TV}}^{(j)}$ is a piecewise constant image.

(d)

Contrast enhancement. Use the stable part of EIT data to modify $\sigma_{\mathrm{TV}}^{(j)}$ into an optimal image called $\sigma_{\mathrm{CE}}^{(j)}$. If $j=J_{\text {iter }}$, then return $\sigma_{\mathrm{CE}}^{(j)}$ and stop.

(e)

$$
\sigma_{\mathrm{CE}}^{(j)}
$$

Define $\mathbf{t}^{j}(k)$ for $R<|k| \leq \widetilde{R}$ as the new transform $\tilde{\mathbf{t}}^{j}(k)$ of $\sigma_{\mathrm{CE}}^{(j)}$, for $|k|<R-1$ as $\mathbf{t}^{0}(k)$, and for $R-1<$ $|k| \leq R$ as a blend of $\mathbf{t}^{0}$ and $\tilde{\mathbf{t}}^{j}$. Set $j:=j+1$.

Figure 3. Flowchart of the proposed edge-preserving EIT reconstruction method.

as used in most of the previous Mumford-Shah-based approaches. The edge enhanced image is further used to recover missing high frequency coefficients in the D-bar procedure. What are the advantages and disadvantages of the proposed approach over iterative optimization algorithms arising from variational regularization of EIT?

- The data-driven contrast enhancement step (see section 5.2) is a very effective component of the proposed method. Implementing such a step in variational regularization 
does not seem to be straightforward.

- The proposed alternation between D-bar reconstruction and TV segmentation keeps the low-frequency part of the reconstruction correct by using the transform data in a disc in the frequency domain.

- Our method is computationally more expensive than variational regularization. However, for the segmentation part, the proposed approach is much faster than previous level set-based methods.

- We use the CGO sinogram (see section 5.1) as the data discrepancy measure. It is more robust than current-voltage measurement matrices typically used in variational regularization, as the sinogram only contains stable information nonlinearly extracted from EIT data. One could use the CGO sinogram as the data model in variational regularization as well but at the expense of computation time.

Finally, we remark that the proposed method differs from [25], where the concept of CGO sinogram was introduced, by instead focusing on enlarging the scattering radius to produce more accurate conductivity reconstructions.

The rest of this paper is organized as follows. In section 2 we formulate the mathematical EIT model and review the relevant literature. Section 3 is devoted to a discussion of the shortcut method. The edge-promoting TV flow is described in section 4. Section 5 introduces a contrast enhancement step based on the CGO sinogram, which is useful as a robust datafidelity term. The new algorithm we present in this work is tested on two discontinuous phantoms for varying levels of noise. Section 6 outlines the computational details, and in section 7 the numerical results are presented and discussed. We conclude our findings in section 8 .

2. Mathematical model and literature review. We concentrate on the two-dimensional case with $\Omega$ representing the unit disc. However, all our techniques can be extended to simply connected domains $\Omega \subset \mathbb{R}^{2}$ with a piecewise smooth Lipschitz boundary $\partial \Omega$. Throughout the paper the following notation is used. For $r>0, D(0, r)$ denotes the disc in the plane centered at the origin with radius $r$. For any open set $U$ in the plane, $\bar{U}$ denotes the closure of $U$. We associate $\mathbb{C}$ and $\mathbb{R}^{2}$ by $z=(x, y)=x+i y$.

Let $f$ denote the electric voltage potential maintained at the boundary. The corresponding potential $u$ inside the domain $\Omega$ satisfies the Dirichlet problem for the elliptic conductivity equation

$$
\begin{aligned}
\nabla \cdot \sigma(z) \nabla u(z) & =0, \quad z \in \Omega, \\
\left.u\right|_{z \in \partial \Omega} & =f(z), \quad z \in \partial \Omega,
\end{aligned}
$$

where $\sigma \in L^{\infty}(\Omega)$ is an isotropic conductivity satisfying $\sigma(z) \geq c>0$. The Dirichlet-toNeumann (D-N) map is defined by

$$
\Lambda_{\sigma} f=\left.\sigma \frac{\partial u}{\partial \nu}\right|_{\partial \Omega}
$$

where $\nu$ denotes the outward facing unit normal vector to the boundary. It is well known that $\Lambda_{\sigma}: H^{1 / 2}(\partial \Omega) \rightarrow H^{-1 / 2}(\partial \Omega)$ is a bounded linear operator. One can think of $\Lambda_{\sigma}$ as a mathematical model for voltage-to-current measurements performed at the boundary. 
The above mathematical model for the inverse conductivity problem was first formulated by Alberto Calderón in 1980 [10]. In practical EIT, one needs to recover the electric conductivity distribution $\sigma: \Omega \rightarrow \mathbb{R}$ in a regularized manner from noisy boundary measurements $\Lambda_{\sigma}^{\delta}$, where $\left\|\Lambda_{\sigma}-\Lambda_{\sigma}^{\delta}\right\|_{Y} \leq \delta$ for a known noise level $\delta$ and an appropriate norm $\|\cdot\|_{Y}$. Among currently available EIT algorithms, the D-bar method is the only one with a regularization analysis complete with a convergence rate [37]. For progress in regularization analysis based on variational approaches, see $[39,32]$. For regularized methods for extracting partial information on $\sigma$, see [30, 27].

The D-bar method is based on a nonlinear Fourier transform, which is not physically measurable. The definition of the transform depends on certain "almost exponential" functions, the so-called complex geometric optics (CGO) solutions. CGO solutions were introduced by Faddeev [24] and later introduced in the context of inverse problems by Sylvester and Uhlmann [51]. As explained below in section 3, we use a regularized D-bar method combining the approaches of [44] and [3, 4]. The components of this nonlinear algorithm were developed in $[50,40,38,36,37,2,1,5]$. For reconstructions from experimental data, see [31, 43, 20, 22, 28]. For a more details about the history of EIT and D-bar methods, see [41, Chapter 14].

\section{The "shortcut" D-bar reconstruction method.}

3.1. The Schrödinger-type D-bar method. The regularized D-bar method $[44,50,37]$ for $C^{2}$-conductivities consists of two steps, namely $\Lambda_{\sigma}^{\delta} \stackrel{1}{\longrightarrow} \mathbf{t}_{R}(k) \stackrel{2}{\longrightarrow} \sigma_{R}(z)$. Step 1 is not used here; we refer the reader to [41, Chapter 15] for details.

Step 2 goes from truncated scattering data $\mathbf{t}_{R}: \mathbb{C} \rightarrow \mathbb{C}$, supported in the disc $|k|<R$, to the regularized conductivity as follows. For each $z \in \Omega$, solve the integral equation

$$
m_{R}(z, k)=1+\frac{1}{(2 \pi)^{2}} \int_{|\kappa|<R} \frac{\mathbf{t}_{R}(\kappa)}{(k-\kappa) \bar{\kappa}} e(-z, \kappa) \overline{m_{R}(z, \kappa)} d \kappa_{1} d \kappa_{2}
$$

where $e(z, k):=\exp \{i(k z+\bar{k} \bar{z})\}=\exp \{2 i \operatorname{Re}(k z)\}$. The regularized conductivity is computed by

$$
\sigma_{R}(z)=\left(m_{R}(z, 0)\right)^{2}
$$

3.2. The Beltrami-type D-bar method. The D-bar method for $L^{\infty}$-conductivities $[3,4,1]$ is based on CGO solutions $f_{ \pm \mu}(z, k)$ to the Beltrami equation

$$
\bar{\partial}_{z} f_{ \pm \mu}(z, k)= \pm \mu(z, k) \overline{\partial_{z} f_{ \pm \mu}(z, k)},
$$

where $\mu(z)=(1-\sigma(z)) /(1+\sigma(z))$ and $f_{ \pm \mu}(z, k)=e^{i k z}\left(1+\mathcal{O}\left(\frac{1}{|z|}\right)\right)$ as $|z| \rightarrow \infty$. Set $M_{ \pm \mu}(z, k)=e^{-i k z} f_{ \pm \mu}(z, k)$. The reconstruction method has three steps:

$$
\left.\Lambda_{\sigma}^{\delta} \stackrel{1}{\longrightarrow} M_{ \pm \mu}(\cdot, k)\right|_{\partial \Omega} \stackrel{2}{\longrightarrow} \underset{\text { Matrix }}{\text { Transport }} \stackrel{3}{\longrightarrow} \sigma_{R}(z)
$$

out of which we only use step 1 (for steps 2 and 3 , see [41, section 16.3]). For each fixed $k \in \mathbb{C}$, $|k|<R$, solve

$$
\left.M_{ \pm \mu}(\cdot, k)\right|_{\partial \Omega}+1=\left.\left(\mathcal{P}_{ \pm \mu}^{k}+\mathcal{P}_{0}\right) M_{ \pm \mu}(\cdot, k)\right|_{\partial \Omega}
$$


to obtain the CGO traces $M_{ \pm \mu}(\cdot, k)$ for $z \in \partial \Omega$, where $\mathcal{P}_{ \pm \mu}^{k}$ and $\mathcal{P}_{0}$ are the projection operators described in [1].

3.3. The shortcut method. There is a connection [2, section 5] between the Beltrami CGOs of $[3,4]$ and Schrödinger CGOs of [44], as well as their associated scattering transforms $\tau(k)$ and $\mathbf{t}(k)$, respectively [2, section 6]. Namely, when $\sigma \in C^{2}$ we have

$$
\mathbf{t}(k)=-4 \pi i \bar{k} \tau(k)=-2 i \bar{k} \int_{\mathbb{R}^{2}} \overline{\left(\bar{\partial}_{z}\left(M_{+\mu}(z, k)-M_{-\mu}(z, k)\right)\right)} d z_{1} d z_{2} .
$$

Numerical evidence [5] suggests that the above connection holds for $\sigma$ with jump discontinuities, resulting in numerical equivalence between the reconstructed conductivities. In fact, the solution of (3.1) is faster and more stable than the transport matrix method of [1]. Therefore, we will use the combined D-bar algorithm presented in [5], called the shortcut method, which has the following steps:

Step 1: From noisy boundary measurements $\Lambda_{\sigma}^{\delta}$ to determine the CGO boundary traces $\left.M_{ \pm \mu}(\cdot, k)\right|_{\partial \Omega}$ by solving $(3.4)$.

Step 2: From boundary CGO traces $\left.M_{ \pm \mu}(\cdot, k)\right|_{\partial \Omega}$ to truncated scattering data $\mathbf{t}_{R}(k)$. The analyticity of $M_{ \pm \mu}(\cdot, k)$ outside $\Omega$ leads to the following development for $|z|>1$ :

$$
M_{ \pm \mu}(z, k)=1+\frac{a_{1}^{ \pm}(k)}{z}+\frac{a_{2}^{ \pm}(k)}{z^{2}}+\cdots
$$

from which one defines $\mathbf{t}_{R}(k):=-4 \pi i \bar{k} \tau_{R}(k)$, where

$$
\tau_{R}(k):= \begin{cases}\frac{1}{2}\left(\overline{a_{1}^{+}(k)}-\overline{a_{1}^{-}(k)}\right), & |k|<R, \\ 0, & |k| \geq R .\end{cases}
$$

Step 3: From truncated scattering data $\mathbf{t}_{R}(k)$ to conductivity $\sigma_{R}(z)$. Solve (3.1) and evaluate $(3.2)$.

4. Image segmentation method. Image segmentation plays an important role in many applications of computer vision and medical image analysis. The objective is to divide the image into constituent subdomains of different characteristics. In this paper, we are interested in representing the conductivity image with piecewise constant components using the celebrated Mumford-Shah approach [42]. For the references on the Mumford-Shah approach and its related works, such as the active contour model [17] using TV regularization [48], one may refer to the monographs $[6,15,8]$.

For simplicity of notation, let $\sigma_{0}(x)$ be the input conductivity image defined on $\Omega$. The image segmentation problem is to find a partition of $\Omega$ into $K$ disjoint subdomains $\left\{\Omega_{k}\right\}_{k=1}^{K}$, i.e.,

$$
\Omega=\bigcup_{k=1}^{K} \Omega_{k} ; \quad \Omega_{k} \cap \Omega_{j}=\emptyset \text { for } k \neq j .
$$

The Mumford-Shah variational segmentation problem is as follows: find a piecewise smooth function and a partition edge (closed) set $\Gamma=\bigcup_{k=1}^{K} \partial \Omega_{k}$ such that the following 
functional is minimized:

$$
E(h, \Gamma)=\frac{\lambda}{2} \int_{\Omega}\left(h(x)-\sigma_{0}(x)\right)^{2} d x+\int_{\Omega \backslash \Gamma}|\nabla h(x)|^{2} d x+\alpha \mathcal{H}^{1}(\Gamma),
$$

where $\mathcal{H}^{1}(\Gamma)$ denotes the one-dimensional Hausdorff measure of the edge set $\Gamma$. Due to the complexity of the model, many simplifications are proposed. In the simplest form of the model, $h$ is assumed to be piecewise constant on each $\Omega_{k}$ and the model is reduced to

$$
\min _{\left\{\Omega_{k}, c_{k}\right\}_{k=1}^{K}}\left\{\frac{\lambda}{2} \sum_{k=1}^{K} \int_{\Omega_{k}}\left(\sigma_{0}(x)-c_{k}\right)^{2} d x+\sum_{k=1}^{K}\left|\partial \Omega_{k}\right|\right\},
$$

where $c_{k} \in \mathbb{R}$ for $k=1, \ldots, K$ is the mean intensity for each subregion $\Omega_{k}$. The parameter $\lambda>0$ is used to balance the data fitting and the total length of regions interfaces. This model (4.1) is hard to solve directly. In fact, when the regions $\Omega_{k}$ are determined, the optimal $c_{k}$ is given as

$$
c_{k}=\frac{\int_{x \in \Omega_{k}} \sigma_{0}(x) d x}{\left|\Omega_{k}\right|} .
$$

Thus we can consider an alternating scheme on solving $\Omega_{k}$ and $c_{k}$ iteratively. Once $c_{k}$ is determined, we solve for the $\Omega_{k}$. By doing so, we introduce the labeling function $u_{k}$ of the disjoint subregions $\Omega_{k}$ :

$$
u_{k}(x)=\left\{\begin{array}{ll}
1 & \text { if } x \in \Omega_{k}, \\
0 & \text { otherwise }
\end{array} \quad \text { for } k=1, \ldots, K .\right.
$$

According to the co-area formula, the perimeter of a set $\Omega_{k}$ is given by the TV of $u_{k}$,

$$
\left|\partial \Omega_{k}\right|=\int_{\Omega}\left|D u_{k}\right|
$$

and the TV $\int_{\Omega}|D u|$ is defined in the distribution sense as

$$
\int_{\Omega}|D u|:=\sup \left\{-\int_{\Omega} u \operatorname{div} \phi d x: \phi \in C_{c}^{\infty}\left(\Omega ; \mathbb{R}^{d}\right),|\phi(x)| \leq 1 \text { a.e. } x \in \Omega\right\} .
$$

It is well known that if $u \in W^{1,1}(\Omega)$, then $\int_{\Omega}|D u|=\int_{\Omega}|\nabla u(x)| d x$.

Meanwhile, since each pixel can only be assigned as one region, the labeling function $u_{k}(x)$ satisfies the following constraint:

$$
\sum_{k=1}^{K} u_{k}(x)=1 \text { a.e. } x \in \Omega .
$$

Generally, convex relaxation is made by allowing $u_{i}$ to take values continuously in $[0,1]$ to overcome the computation complexity of the binary constraint. The overall model is reformulated as

$$
\min _{\left\{u_{k}, c_{k}\right\}_{k=1}^{K}}\left\{\sum_{k=1}^{K} \int_{\Omega}\left|D u_{k}\right|+\sum_{k=1}^{K} \int_{\Omega} u_{k} h_{k}\right\} \text { s.t. }\left(u_{1}(x), \ldots, u_{K}(x)\right) \in \boldsymbol{S},
$$


where we denote

$$
h_{k}(x)=\frac{\lambda}{2}\left(\sigma_{0}(x)-c_{k}\right)^{2}
$$

and the constraint set

$$
\boldsymbol{S}=\left\{\left(u_{1}, \ldots, u_{K}\right) \in \operatorname{BV}\left(\Omega,[0,1]^{K}\right): \sum_{k=1}^{K} u_{k}(x)=1 \text { a.e. } x \in \Omega\right\},
$$

and $\operatorname{BV}\left(\Omega,[0,1]^{K}\right)$ denotes the bounded variation functions product space valued on $[0,1]^{K}$.

With fixed $c_{k}$, the convex relaxed formulation for $u_{k}$ allows us to develop efficient algorithms based on the well-studied TV minimization. For example, it has been extensively studied in $[13,14,7,46,11]$. Theoretically, the global solution to the original binary model can be achieved for the case of two regions when the intensities $c_{k}$ are given.

This above region-based model can be further combined with an edge-based approach to improve the segmentation quality and speed, such as in $[49,9,7]$. Assuming $u_{k} \in W^{1,1}(\Omega)$, the weighted TV model can be defined as

$$
J(\boldsymbol{u})=\sum_{k=1}^{K} \int_{\Omega} g(x)\left|\nabla u_{k}(x)\right| d x
$$

where $g(x) \geq 0$ is an edge function taking small values at locations with a large gradient and large values for a smooth region. For example, a usual choice is

$$
g(x)=\frac{1}{1+s\left\|\nabla \tilde{\sigma_{0}}(x)\right\|^{2}},
$$

where $\tilde{\sigma_{0}}$ is a smoothed version of the given image $\sigma_{0}$ and $s>0$ is a positive number. Note that if $g(x)$ is identical to 1 , it reduces to the model (4.7).

In the following, we present a primal-dual splitting method used in [46, 23, 12, 52]. Denote $\boldsymbol{u}=\left(u_{1}, \ldots, u_{K}\right)$ and $\boldsymbol{h}=\left(h_{1}, \ldots, h_{K}\right)$, and denote $J(\boldsymbol{u})=\sum_{k=1}^{K} \int_{\Omega} g(x)\left|\nabla u_{k}\right| d x$ and $\langle\boldsymbol{u}, \boldsymbol{h}\rangle=\sum_{k=1}^{K} \int_{\Omega} u_{k}(x) h_{k}(x) d x$; then the convex minimization problem is rewritten as

$$
\boldsymbol{u}^{*}=\arg \min _{\boldsymbol{u} \in \boldsymbol{S}}\{J(\boldsymbol{u})+\langle\boldsymbol{u}, \boldsymbol{h}\rangle\} .
$$

Based on the dual definition (4.5), we consider the following min-max model:

$$
\min _{\boldsymbol{u} \in \boldsymbol{S}} \max _{\boldsymbol{p} \in \boldsymbol{T}} E(\boldsymbol{u}, \boldsymbol{p})=\{\langle\boldsymbol{u}, \operatorname{div}(\boldsymbol{p})\rangle+\langle\boldsymbol{u}, \boldsymbol{h}\rangle\},
$$

where $\boldsymbol{p}=\left(p_{1}, \ldots, p_{K}\right)$ for $p_{k} \in C_{c}^{\infty}\left(\Omega ; \mathbb{R}^{d}\right),\langle\boldsymbol{u}, \operatorname{div}(\boldsymbol{p})\rangle=\sum_{k=1}^{K} \int_{\Omega} u_{k}(x) \operatorname{div}\left(p_{k}(x)\right) d x$, and

$$
\boldsymbol{T}=\left\{\boldsymbol{p}=\left(p_{1}, \ldots, p_{K}\right):\left(\sum_{i=1}^{d}\left|p_{k}^{d}(x)\right|^{2}\right)^{1 / 2} \leq g(x) \text { a.e. } x \in \Omega \text { and for all } k=1, \ldots, K\right\} \text {. }
$$

The specific algorithm is given as follows: 
Step 0: Initialization: Choose $c_{1}, \ldots, c_{K}$ as the initial guess of the mean intensity of each region. $\tau_{1}, \tau_{2}>0$ are parameters such that $\tau_{1} \tau_{2} \leq 1 / 8$ and $\left(\boldsymbol{p}^{0}, \boldsymbol{u}^{0}\right) \in \boldsymbol{T} \times \boldsymbol{S}$. $\overline{\boldsymbol{u}}^{0}=\boldsymbol{u}^{0}$.

Set $i:=0$ and run the outer loop as follows:

Step 1: Set $j:=0$ and run the inner loop to compute $u_{k}(x)$ for $k=1, \ldots, K$ and $x \in \Omega$.

Step 1.1: Compute the dual variable $\boldsymbol{p}^{j+1}=\Pi_{\boldsymbol{T}}\left(\boldsymbol{p}^{j}+\tau_{1} \nabla \overline{\boldsymbol{u}}^{j}\right)$, where $\Pi_{\boldsymbol{T}}(\cdot)$ denotes the projection operator onto the convex set $\boldsymbol{T}$.

Step 1.2: Compute the primal variable $\boldsymbol{u}^{j+1}=\Pi_{\boldsymbol{S}}\left(\boldsymbol{u}^{j}+\tau_{2}\left(\boldsymbol{h}_{i}+\operatorname{div} \boldsymbol{p}^{j+1}\right)\right)$, where $\Pi_{\boldsymbol{S}}(\cdot)$ denotes the projection operator onto the convex set $\boldsymbol{S}$.

Step 1.3: Compute the auxiliary primal variable: $\overline{\boldsymbol{u}}^{j+1}=\boldsymbol{u}^{j+1}+\left(\boldsymbol{u}^{j+1}-\boldsymbol{u}^{j}\right)$.

Step 1.4: Set $j=j+1$, update until the stopping conditions are satisfied, and output $\boldsymbol{u}$.

Step 2: Compute the piecewise regions $\Omega_{k}$ by the binarization of $u_{k}(x)$. Generally, a global minimizer of (4.12) might not be binary, and a final thresholding step needs to be taken to get a binary solution

$$
u_{k}^{*}(x)= \begin{cases}1 & \text { if } u_{k}^{*}(x)=\max \left\{u_{1}(x), u_{2}(x), \ldots, u_{K}(x)\right\} \\ 0 & \text { otherwise. }\end{cases}
$$

If the maximizer is not unique, the maximizer with the smallest subscript is used as a convention.

Step 3: Update the mean intensity estimation: $c_{k}^{i}$ for $k=1, \ldots, K$ by (4.2) and $\boldsymbol{h}_{i}$ by (4.8).

Step 4: Set $i=i+1$ and update until the stopping conditions are satisfied.

After we obtain the label functions $u_{k}^{*}(x)$ and $c_{k}$ for $k=1, \ldots, K$, an image can be reconstructed as a piecewise constant function with the mean intensity $c_{k}$ in the corresponding $k$ th region, i.e.,

$$
\sigma_{T V}(x)=\sum_{k=1}^{K} u_{k}^{*}(x) c_{k}
$$

Hence, this reconstructed image can be used as a piecewise constant regularized approximation to the original image by letting $\sigma_{0}(x):=\sigma_{D B}(x)$ in the whole algorithm described in Figure 3.

\section{Data-driven contrast adjustments.}

5.1. The Beltrami CGO sinogram. We extend the concept of the CGO sinogram, introduced in [25], to discontinuous conductivities. Set

$$
\mathcal{S}_{\sigma}(\theta, \varphi, \rho):=M_{\mu}\left(e^{i \theta}, \rho e^{i \varphi}\right)-1,
$$


where $z=e^{i \theta}$ and $k=\rho e^{i \varphi}$ for $\theta, \varphi \in[-\pi, \pi)$ and the traces $M_{\mu}\left(e^{i \theta}, \rho e^{i \varphi}\right)$ of the CGO solutions are determined from the noisy EIT data $\Lambda_{\sigma}^{\delta}$ using (3.4). The radius $\rho$ must be smaller than the noise-dependent cutoff frequency $R$.

The traditional data-fidelity term used in EIT is $\left\|\Lambda_{\sigma}-\Lambda_{\sigma^{\prime}}\right\|$. We use instead the CGO sinogram data-fidelity term

$$
\left\|\mathcal{S}_{\sigma}(\theta, \varphi, \rho)-\mathcal{S}_{\sigma^{\prime}}(\theta, \varphi, \rho)\right\|_{L^{2}\left(\mathbb{T}^{2}\right)}^{2},
$$

where $\mathbb{T}^{2}$ denotes the two-dimensional torus.

5.2. Contrast enhancement. Assume that the piecewise constant conductivity satisfies $\operatorname{supp}(\sigma-1) \subset \Omega$ and that we know a priori approximate bounds $0<c<1$ and $C>1$ such that

$$
\min _{z \in \Omega} \sigma(z)>c, \quad \max _{z \in \Omega} \sigma(z)<C .
$$

Let $\widetilde{\sigma}$ denote an approximate reconstruction to the true conductivity $\sigma$ defined on $\Omega$, whose contrast we intend to improve, and $\operatorname{suppose} \operatorname{supp}(\widetilde{\sigma}-1) \subset \Omega$. Set $f(z)=\widetilde{\sigma}-1$ and denote

$$
m=\min _{z \in \Omega} f(z), \quad M=\max _{z \in \Omega} f(z),
$$

and assume that $m<0$ and $M>0$. Let $s$ and $t$ be two parameters such that $0 \leq s \leq 1$ and $0 \leq t \leq 1$ and define

$$
\sigma_{s, t}(z):=1+ \begin{cases}t(C-1) f(z) / M & \text { for } z \text { satisfying } \tilde{\sigma}(z)>1 \\ s(c-1) f(z) / m & \text { for } z \text { satisfying } \tilde{\sigma}(z)<1 \\ 0 & \text { otherwise. }\end{cases}
$$

Note that $\sigma_{0,0} \equiv 1$, and the maximum values $s=1$ and $t=1$ yield the maximal image contrast below and above 1 , respectively, i.e.,

$$
\min _{z \in \Omega} \sigma_{1, t}(z)=c, \quad \max _{z \in \Omega} \sigma_{s, 1}(z)=C .
$$

We determine the optimal values for $s, t$ as the minimizers of the nonlinear data-discrepancy functional based on the CGO sinogram:

$$
\left(s_{0}, t_{0}\right):=\underset{(s, t) \in[0,1]^{2}}{\arg \min } \frac{\left\|\mathcal{S}_{\sigma_{s, t}}(\cdot, \cdot, \rho)-\mathcal{S}_{\sigma}^{\delta}(\cdot, \cdot, \rho)\right\|_{L^{2}\left(\mathbb{T}^{2}\right)}}{\left\|\mathcal{S}_{\sigma}^{\delta}(\cdot, \cdot, \rho)\right\|_{L^{2}\left(\mathbb{T}^{2}\right)}} .
$$

The result of contrast enhancement is then $\sigma_{\mathrm{CE}}:=\sigma_{s_{0}, t_{0}}$.

In this paper, the objective function in (5.5) is minimized via the DIRECT algorithm [45], in a fashion analogous to [25]. The expression DIRECT refers to "DIviding RECTangles," which suggests the strategy of this sampling, global search algorithm.

\section{Numerical implementation.}

6.1. Simulation of noisy EIT data. Our numerical experiments deal with two simulated discontinuous conductivity phantoms, namely a heart-and-lungs phantom $\sigma_{1}$ and a crosssection of a stratified oil pipeline phantom $\sigma_{2}$. See Table 1 and Figure 4 . 
Table 1

Conductivity values in the two simulated phantoms shown in Figure 4. Left: heart-and-lungs phantom $\sigma_{1}$. Right: oil pipeline phantom $\sigma_{2}$.

\begin{tabular}{|ll|ll|}
\hline$\sigma_{1}$ & & $\sigma_{2}$ & \\
\hline Background & 1.0 & Pipe & 1.0 \\
Lung & 0.5 & Top layer (oil) & 1.2 \\
Heart & 2.0 & Middle layer (water) & 2.0 \\
& & Bottom layer (sand) & 0.3 \\
\hline
\end{tabular}
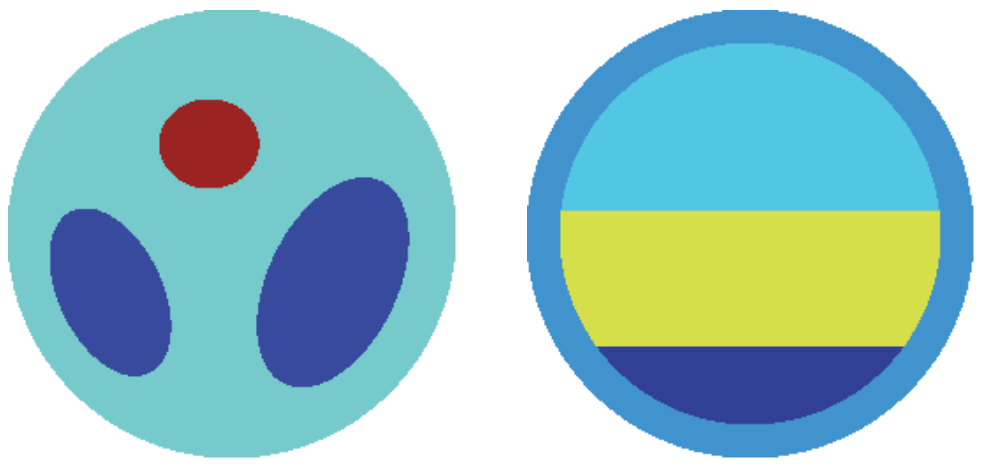

Figure 4. True conductivity phantoms; for conductivity values, see Table 1. Left: heart-and-lungs phantom $\sigma_{1}$. Right: pipeline phantom $\sigma_{2}$.

6.1.1. Computation of the discrete Neumann-to-Dirichlet map. EIT data was simulated using the finite element method and following [41, sections 13.2.3 and 16.3.3]. We use the trigonometric basis functions

$$
\phi_{n}(\theta)= \begin{cases}\pi^{-1 / 2} \cos ((n+1) \theta / 2) & \text { for odd } n, \\ \pi^{-1 / 2} \sin (n \theta / 2) & \text { for even } n,\end{cases}
$$

where $1 \leq n \leq 2 N$. The Neumann-to-Dirichlet map $\mathcal{R}_{\sigma}$ is approximated by the matrix $\mathbf{R}_{\sigma}=\left[\left(\mathbf{R}_{\sigma}\right)_{m, n}\right]$ given by

$$
\left(\mathbf{R}_{\sigma}\right)_{m, n}=\left\langle\mathbf{R}_{\sigma} \phi_{n}, \phi_{m}\right\rangle=\int_{0}^{2 \pi}\left(\mathbf{R}_{\sigma} \phi_{n}\right)(\theta) \phi_{m}(\theta) d \theta
$$

where $\mathbf{R}_{\sigma} \phi_{n}=\left.u_{n}\right|_{\partial \Omega}$ with $\nabla \cdot \sigma \nabla u_{n}=0$ in $\Omega,\left.\left(\sigma\left(\partial u_{n} / \partial \nu\right)\right)\right|_{\partial \Omega}=\phi_{n}$, and $\int_{\partial \Omega} u_{n} d S=0$. Here, $1 \leq m, n \leq 2 N$. In this paper, we use $N=16$, which corresponds to 33 linearly independent current patterns.

Relative Gaussian noise was added to the boundary voltage data as in [26]. Namely, define $\widetilde{\mathbf{R}}_{\sigma}$ with $\left(\widetilde{\mathbf{R}}_{\sigma}\right)_{m, n}=\left\langle\widetilde{\mathbf{R}}_{\sigma} \phi_{n}, \phi_{m}\right\rangle$, where

$$
\widetilde{\mathbf{R}}_{\sigma} \phi_{n}=\mathbf{R}_{\sigma} \phi_{n}+\eta \mathcal{N}_{n}\left\|\mathbf{R}_{\sigma} \phi_{n}\right\|_{L^{\infty}}
$$

$\eta$ denotes the noise level so that $100 \eta \%$ noise is added, and $\mathcal{N}_{1}, \ldots, \mathcal{N}_{2 N+1}$ are independent Gaussian distributions with mean zero and variance one, which are implemented through the MATLAB function randn generating pseudorandom values drawn from the standard normal 
distribution $\mathcal{N}(0,1)$. The noisy D-N matrix is then formed by inverting $\widetilde{\mathbf{R}}_{\sigma}$ and adding a zero row and column on the top and left as described in [41].

The algorithm outlined in Figure 3 was applied to the two phantoms shown in Figure 4. In this paper, the algorithm was performed for $J_{\text {iter }}=3$ iterations and for three noise levels as follows: zero added noise, $0.1 \%$ added noise, and $0.75 \%$ added noise (corresponding to setting $\eta=0,0.001,0.0075)$. The output of the algorithm after the $J_{\text {iter }}=3$ iterations is denoted by $\sigma_{\mathrm{CE}}^{\left(J_{\text {iter }}\right)}$.

6.2. Computational grids. All the computations on the $z$-plane (D-bar reconstructions, TV flow outputs, and CE outputs) were generated on a $z$-grid of $2^{\ell} \times 2^{\ell}$ equidistributed points of the square $[-s, s) \times[-s, s)$ with $\ell=8$ and $s=2.3$. Thus, the $z$-grid consists of $2^{16}=65536$ points. The D-bar equation solver (used to solve (3.1)) was executed just on the set of 9729 points of the $z$-grid belonging to the closed disc $\bar{\Omega}$. Note that the larger $z$-region is needed to extend the scattering data.

The $k$-grids are problem specific; i.e., for lower levels of noise, a larger radius $R$ can be used for the initial low-pass filtering in the nonlinear Fourier domain. In each case, we fixed the two parameters $R$ (the initial low-pass filtering chosen intuitively by looking at where the scattering data "blows up" in magnitude) and $\widetilde{R}$ (the increased scattering radius used when solving the Beltrami equation). In each case, the $k$-grid for the scattering data was comprised of $2^{7} \times 2^{7}$ equispaced points on the square $[-\widetilde{R}, \widetilde{R}) \times[-\widetilde{R}, \widetilde{R}$ ). The traditional (and original) D-bar image $\sigma_{\mathrm{DB}}^{(1)}$ is computed from scattering data satisfying $|k| \leq R$, and all subsequent D-bar images $\sigma_{\mathrm{DB}}^{(2)}, \sigma_{\mathrm{DB}}^{(3)}$, etc., are computed using the larger disc $|k| \leq \widetilde{R}$.

Remark. If $\operatorname{supp}\left(\sigma-\sigma_{0}\right) \subset D(0,1)$ for some positive constant $\sigma_{0} \neq 1$, the algorithm can be rescaled as follows. Defining $\widetilde{\sigma}:=\sigma / \sigma_{0}$, we have $\operatorname{supp}(\widetilde{\sigma}-1) \subset D(0,1)$. Apply the above algorithm to $\Lambda_{\widetilde{\sigma}}=\sigma_{0} \Lambda_{\sigma}$ and write $\widetilde{\sigma}_{\mathrm{CE}}^{(J)}$ for the output generated. Take $\sigma_{0} \widetilde{\sigma}_{\mathrm{CE}}^{(J)}$ as the final approximation to $\sigma$.

6.3. Computation of the initial nonlinear Fourier transform. This step corresponds to Figure 3(a). From the boundary measurements $\Lambda_{\sigma}^{\delta}$ we use (3.4) and (3.6) to compute the initial scattering data $\tau^{0}(k)$ on a $k$-disc of radius $R$. We write $\mathbf{t}^{0}(k):=-4 \pi i \bar{k} \tau^{0}(k)$.

6.4. D-bar reconstruction. This step corresponds to Figure 3(b). Use the shortcut method described in section 3.3 to compute $\sigma_{\mathrm{DB}}^{(j)}$ from the scattering transform $\mathbf{t}^{j-1}(k)$. If $j=1$, then use the smaller cutoff disc $|k|<R$; else use larger disc $|k|<\widetilde{R}$.

6.5. Edge enhancement using TV flow. This step corresponds to Figure 3(c). Introduce edges into the D-bar image $\sigma_{\mathrm{DB}}^{(j)}$ by applying the segmentation flow of section 4 . The resulting piecewise constant image, defined by (4.14), is called $\sigma_{\mathrm{TV}}^{(j)}$. Note that the initial guess of the mean intensity is obtained directly by the $K$-means algorithm, where $K$ denotes the number of regions preselected. In practice, this is a reasonable guess of the number of regions of different conductivity in one's domain.

6.6. Contrast enhancement. This step corresponds to Figure 3(d). The CGO sinograms were implemented via $33 \times 33$ matrices $\left[(\mathbf{S})_{m, l}\right]$ with $(\mathbf{S})_{m, l}=M_{\mu}\left(z_{m}, k_{l}\right)-1=$ 
$M_{\mu}\left(e^{i \theta_{m}}, 2 e^{i \varphi_{l}}\right)-1$, where

$$
\theta_{m}=(m-1-N) 2 \pi /(2 N+1), \quad 1 \leq m \leq 2 N+1,
$$

with $N=16, \varphi_{l}=\theta_{l}$, and $M_{\mu}(z, k)$ referring to the solution explained in section 5.1 for both the true $\sigma$ and the corresponding approximations $\sigma_{s, t}$. Therefore, $\left\{\theta_{m}\right\}$ and $\left\{\varphi_{l}\right\}$ are the same partition of the interval $(-\pi, \pi)$. Note that one can choose the points $\varphi_{l}$ independently of the $\theta$ values if desired.

Finally, the output image $\sigma_{\mathrm{CE}}^{(j)}$ is determined by plugging $\widetilde{\sigma}=\sigma_{\mathrm{TV}}^{(j)}$ into $(5.4)$ for $\left(s_{0}, t_{0}\right) \in$ $[0,1]^{2}$ obtained via the DIRECT optimization strategy (see (5.5)).

6.7. Extension of the scattering transform. This step corresponds to Figure $3(\mathrm{e})$. The radius of the admissible scattering data is increased from $R$ to $\tilde{R}$ by computing new stable scattering data in the annulus $R-1<|k|<\tilde{R}$ corresponding to the TV-sharpened and contrast-adjusted image $\sigma_{\mathrm{CE}}^{(j)}$ as follows. First, evaluate the Beltrami coefficient $\mu(z)=(1-$ $\left.\sigma_{\mathrm{CE}}^{(j)}(z)\right) /\left(1+\sigma_{\mathrm{CE}}^{(j)}(z)\right)$ on the $z$-grid $[-2.3,2.3) \times[-2.3,2.3)$. Next, solve the Beltrami equation (3.3) for the CGO solutions $f_{ \pm \mu}(z, k)$ for $k$ in $k$-annulus $R-1 \leq|k| \leq \widetilde{R}$. Finally, evaluate the scattering data $\tau(k)$ in the $k$-annulus $R-1 \leq|k| \leq \widetilde{R}$ via

$$
\overline{\tau(k)}:=\frac{1}{2 \pi} \int_{\mathbb{R}^{2}}\left(\bar{\partial}_{z}\left(M_{+\mu}(z, k)-M_{-\mu}(z, k)\right)\right) d z_{1} d z_{2},
$$

where $M_{ \pm \mu}(z, k)=e^{-i k z} f_{ \pm \mu}(z, k)$. Call this new scattering data $\tilde{\tau}^{(j)}(k)$. The new scattering data on the larger radius is then

$$
\tau^{(j)}(k):=\chi(k) \tau^{0}(k)+(1-\chi(k)) \tilde{\tau}^{(j)}(k), \quad|k|<\tilde{R},
$$

where we used the polynomial radial cutoff function $\chi$ defined by

$$
\chi(k)= \begin{cases}1 & \text { if }|k|<R-1, \\ p(|k|-(R-1)) & \text { if } R-1<|k|<R, \\ 0 & \text { if }|k|>R,\end{cases}
$$

with $p(t)=1-3 t^{2}+2 t^{3}$ to blend the data in the overlap region $R-1 \leq|k| \leq R$. Note that in Figure 3 we use the notation $\mathbf{t}^{j}(k):=-4 \pi i \bar{k} \tau^{(j)}(k)$ and $\tilde{\mathbf{t}}^{j}(k):=-4 \pi i \bar{k} \tilde{\tau}^{(j)}(k)$.

6.7.1. Computation of "true" scattering data. We need a comparison for our new extended scattering data with the best possible scattering data. By best possible scattering data we refer to the scattering data that is obtained by computing the CGO solutions to the Beltrami equation (3.3) with $\mu$ corresponding to the true $\sigma$ and by evaluating the scattering data $\tau_{\mathrm{B}}(k)$ via $(6.2)$. For details on how to solve the Beltrami equation and generate the scattering data $\tau_{\mathrm{B}}(k)$, the reader is referred to $[2,5,29]$.

7. Numerical results. The algorithm was tested on the two phantoms shown in Figure 4, and the results are shown here. 
7.1. Example 1: A heart-and-lungs phantom. For the heart-and-lungs phantom, $\sigma_{1}$, it was assumed known a priori that the internal conductivity for this application was bounded between $c=0.3$ and $C=2.5$. Setting the initial scattering radius $R$ to 5 and the enlarged radius $\widetilde{R}$ to 10 for all noise levels proved sufficient. The initial scattering data was reliable for all noise levels within the $k$-disc of radius 5 . The parameters for the TV flow were $K=4$ and $\lambda=0.1$.

The scattering data for each noise level is displayed in Figure 5. The figures contain images of the actual Beltrami scattering transform $\tau_{\mathrm{B}}$ on the larger $k$ disc of radius 10 . This is used to evaluate the efficacy of the new proposed approach. The true scattering data $\tau_{\mathrm{B}}$ was computed by solving (3.3) and (6.2) with the known $\mu=\frac{1-\sigma_{1}}{1+\sigma_{1}}$. It is to serve as a best case scenario baseline.

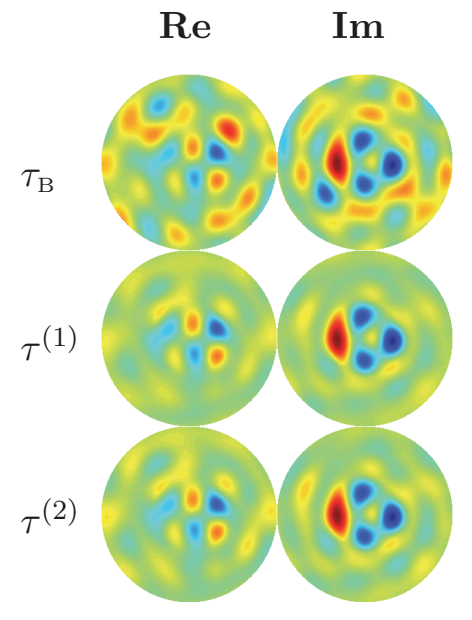

a) no added noise

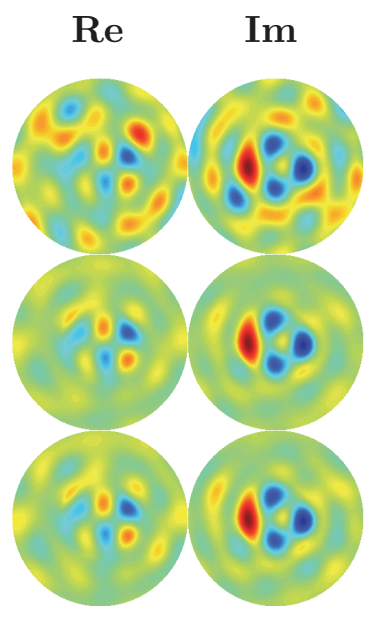

b) $0.1 \%$ noise

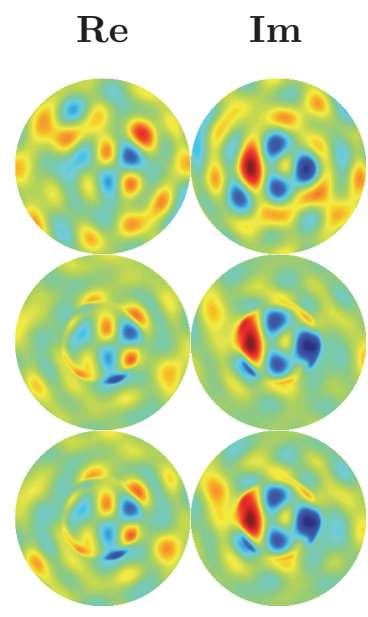

c) $0.75 \%$ noise

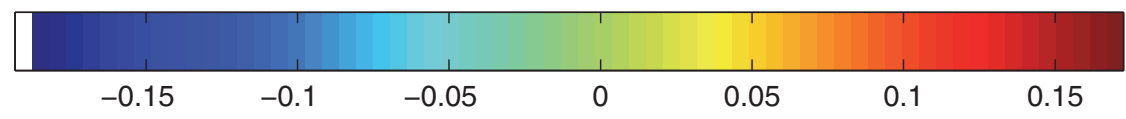

Figure 5. Images of the real and imaginary parts of the "true" scattering transform $\tau_{B}$ (computed directly from the Beltrami equation) and the combined scattering data for the two iterations $\tau^{(1)}$ and $\tau^{(2)}$ from the simulated $D$ - $N$ data corresponding to the heart-and-lungs phantom $\sigma_{1}$.

The reconstructed conductivities for each stage of the algorithm are displayed in Figure 6. Note that the reconstructions are displayed on the same color scale as the original conductivity shown in Figure 4(left) for ease of comparison.

Tables 2, 3, and 4 show the relative $L^{2}$ errors and the Structural SIMilarity (SSIM) index values for the heart-and-lungs phantom $\sigma_{1}$ of Example 1 for zero added noise, $0.1 \%$ added noise, and $0.75 \%$ added noise, respectively. The error values are presented for each step of the proposed algorithm: the D-bar reconstruction $\sigma_{\mathrm{DB}}^{(j)}$, the sharpened reconstruction $\sigma_{\mathrm{TV}}^{(j)}$, and the contrast-adjusted sharpened reconstruction $\sigma_{\mathrm{CE}}^{(j)}$. 


\section{True $\sigma_{1}$}

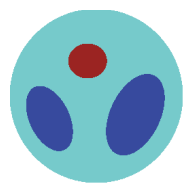

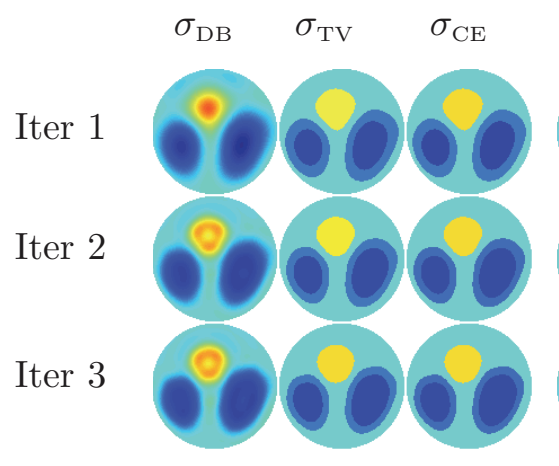

a) no added noise

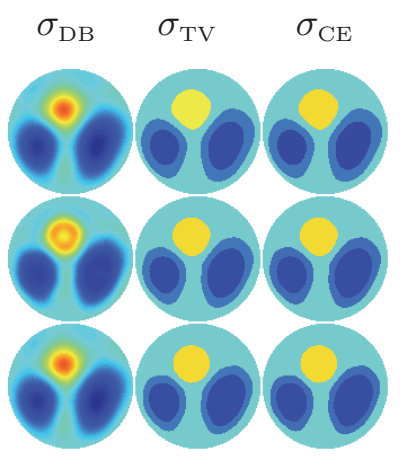

b) $0.1 \%$ noise

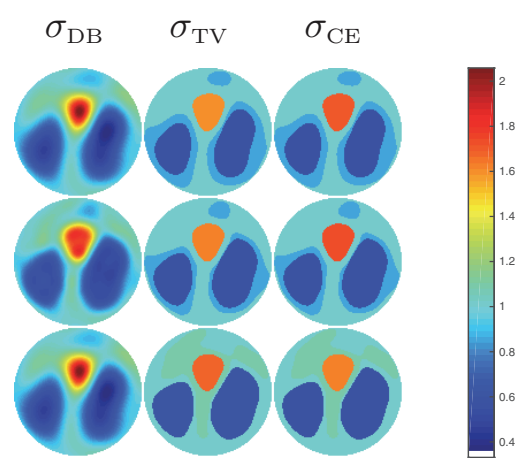

c) $0.75 \%$ noise

Figure 6. This figure shows the real parts of the numerical approximations to the conductivity obtained for the heart-and-lungs phantom of Example 1, i.e., $\sigma_{1}$. The picture consists of three parts (a)-(c) corresponding to our three cases of added simulated noise in the EIT voltage data: zero added noise, noise of relative amplitude $0.1 \%$, and noise of relative amplitude $0.75 \%$, respectively. For the sake of comparison, the true conductivity $\sigma_{1}$ is displayed above the reconstructions (all on the same color scale). For each noise level, the D-bar reconstruction $\sigma_{D B}^{(j)}$ (left columns), the TV sharpened image $\sigma_{T V}^{(j)}$ (middle columns), and the contrast-adjusted TV sharpened images $\sigma_{C E}^{(j)}$ are shown. The first, second, and third rows correspond to the first, second, and third iterations, respectively.

Table 2

The $L^{2}$ relative errors as well as the SSIM values for the zero added noise case for Example 1 with the heart-and-lungs phantom $\sigma_{1}$.

$L^{2}$ Relative Error

\begin{tabular}{l|lll}
\hline$j$ & $\sigma_{\mathrm{DB}}^{(j)}$ & $\sigma_{\mathrm{TV}}^{(j)}$ & $\sigma_{\mathrm{CE}}^{(j)}$ \\
\hline 1 & 0.1240 & 0.1240 & 0.1202 \\
2 & 0.1095 & 0.1185 & 0.1168 \\
3 & 0.1054 & 0.1157 & 0.1145 \\
\hline
\end{tabular}

SSIM

\begin{tabular}{l|lll}
\hline$j$ & $\sigma_{\mathrm{DB}}^{(j)}$ & $\sigma_{\mathrm{TV}}^{(j)}$ & $\sigma_{\mathrm{CE}}^{(j)}$ \\
\hline 1 & 0.6600 & 0.6600 & 0.6541 \\
2 & 0.7351 & 0.6903 & 0.6878 \\
3 & 0.7425 & 0.7117 & 0.7096 \\
\hline
\end{tabular}

In the case of zero and $0.1 \%$ added relative noise, the $L^{2}$ relative error decreases with each iteration and the SSIM value increases. Both measures confirm that the image is "improving." In the case of $0.75 \%$ added relative noise, the $L^{2}$ relative error and SSIM remain approximately the same, but visually one can see the improvements in each iteration, as the artifact present in iterations 1 and 2 (above the heart) is absent in iteration 3. 


\section{Table 3}

The $L^{2}$ relative errors as well as the SSIM values for the $0.1 \%$ added noise case for Example 1 with the heart-and-lungs phantom $\sigma_{1}$.

$L^{2}$ Relative Error

\begin{tabular}{l|lll}
\hline$j$ & $\sigma_{\mathrm{DB}}^{(j)}$ & $\sigma_{\mathrm{TV}}^{(j)}$ & $\sigma_{\mathrm{CE}}^{(j)}$ \\
\hline 1 & 0.1009 & 0.1233 & 0.1194 \\
2 & 0.1083 & 0.1174 & 0.1158 \\
3 & 0.1009 & 0.1142 & 0.1133 \\
\hline
\end{tabular}

SSIM

\begin{tabular}{l|lll}
\hline$j$ & $\sigma_{\mathrm{DB}}^{(j)}$ & $\sigma_{\mathrm{TV}}^{(j)}$ & $\sigma_{\mathrm{CE}}^{(j)}$ \\
\hline 1 & 0.7304 & 0.6603 & 0.6545 \\
2 & 0.7348 & 0.6907 & 0.6883 \\
3 & 0.7304 & 0.7131 & 0.7111 \\
\hline
\end{tabular}

Table 4

The $L^{2}$ relative errors as well as the SSIM values for the $0.75 \%$ added noise case for Example 1 with the heart-and-lungs phantom $\sigma_{1}$.

$L^{2}$ Relative Error

\begin{tabular}{l|lll}
\hline$j$ & $\sigma_{\mathrm{DB}}^{(j)}$ & $\sigma_{\mathrm{TV}}^{(j)}$ & $\sigma_{\mathrm{CE}}^{(j)}$ \\
\hline 1 & 0.1092 & 0.1202 & 0.1167 \\
2 & 0.1076 & 0.1165 & 0.1134 \\
3 & 0.1092 & 0.1151 & 0.1171 \\
\hline
\end{tabular}

SSIM

\begin{tabular}{l|lll}
\hline$j$ & $\sigma_{\mathrm{DB}}^{(j)}$ & $\sigma_{\mathrm{TV}}^{(j)}$ & $\sigma_{\mathrm{CE}}^{(j)}$ \\
\hline 1 & 0.6897 & 0.6680 & 0.6639 \\
2 & 0.6964 & 0.6816 & 0.6776 \\
3 & 0.6897 & 0.6773 & 0.6790 \\
\hline
\end{tabular}

7.2. Example 2: An industrial pipeline. For the cross-section of the industrial pipeline phantom, $\sigma_{2}$, it was assumed known a priori that the internal conductivity for this application was bounded between $c=0.1$ and $C=2.5$. Here we used $K=5$ in the TV flow, and Table 5 gives the values of the scattering radii and the $\lambda$ parameter used in the TV flow for each noise level. Similarly to Example 1, Figure 7 displays the scattering data for each noise level on the larger $k$ disc of radii of 10, 8.3, 6.6 with the "true" Beltrami scattering transform $\tau_{\mathrm{B}}$ included to evaluate the efficacy of the new proposed approach.

\section{Table 5}

Parameter values for the new algorithm for each noise level for the industrial pipeline phantom $\sigma_{2}$.

\begin{tabular}{|l|l|l|l|}
\hline Added noise level & $0 \%$ & $0.1 \%$ & $0.75 \%$ \\
\hline \hline$R$ & 6 & 5 & 4 \\
\hline$\widetilde{R}$ & 10 & 8.3 & 6.6 \\
\hline$\lambda$ & 0.3 & 0.5 & 0.5 \\
\hline
\end{tabular}

The reconstructed conductivities for each stage of the algorithm are displayed in Figure 8. Note that the reconstructions are displayed on the same color scale as the original conductivity shown in Figure 4(right) for ease of comparison. Furthermore, note that the ring of constant conductivity along the boundary (representing the thickness of the pipe) has been enforced in the reconstructions, as this can also be considered a priori information for this application.

Tables 6,7 , and 8 show the relative $L^{2}$ errors and the SSIM values for the industrial pipe phantom $\sigma_{2}$ of Example 2 for zero added noise, $0.1 \%$ added noise, and $0.75 \%$ added noise, respectively. The error values are presented for each step of the proposed algorithm: the D-bar reconstruction $\sigma_{\mathrm{DB}}^{(j)}$, the sharpened reconstruction $\sigma_{\mathrm{TV}}^{(j)}$, and the contrast-adjusted sharpened reconstruction $\sigma_{\mathrm{CE}}^{(j)}$. 


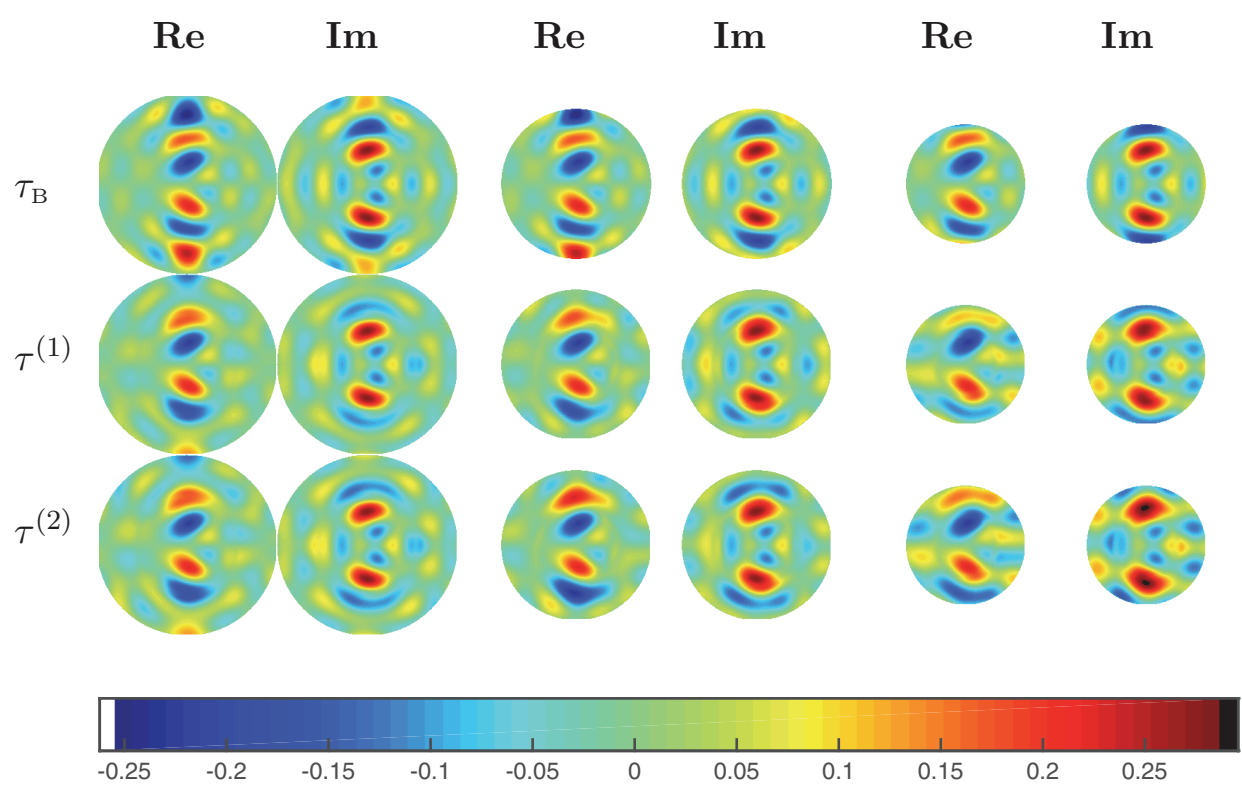

Figure 7. Images of the real and imaginary parts of the "true" scattering transform $\tau_{B}$ (computed directly from the Beltrami equation) and the combined scattering data for the two iterations $\tau^{(1)}$ and $\tau^{(2)}$ from the simulated $D-N$ data corresponding to the industrial pipeline phantom $\sigma_{2}$. Left: zero added noise and $\tilde{R}=10$. Middle: $0.1 \%$ added noise and $\tilde{R}=8.3$. Right: $0.75 \%$ added noise and $\tilde{R}=6.6$.

In the zero added noise case, we see that the $L^{2}$ relative error in the D-bar images decreases with each iteration and the SSIM stays approximately the same. Interestingly, the sharpened and contrast-adjusted images appear to perform slightly worse under the metrics. However, visually the sharpened images reflect the physical scenario (oil, water, sand) much better than their smooth D-bar counterparts, as they contain nice clean divisions between the layers. As the noise level increases, the method is still able to clearly distinguish between oil, water, and sand at even the first iteration, and thus the algorithm could be stopped there (i.e., at $\sigma_{\mathrm{TV}}^{(1)}$ ) for each noise level.

Regarding the contrast-enhanced images, recall that the approximate upper and lower bounds $C=2.5$ and $c=0.1$, respectively, were used. In practice, even closer approximations may be known (in particular for the case of oil, water, and sand), therefore improving the reconstructed values. An investigation into the optimal parameters $c$ and $C$ and the minimization scheme for the contrast adjustment is outside the scope of this introductory paper.

8. Conclusions. EIT data contains information about the conductivity in an indirect, nonlinear, and unstable way. Theoretically, [4] shows that infinite-precision data (the D-N map) contains enough information to uniquely determine the conductivity. However, a practical data matrix $\Lambda_{\sigma}^{\delta}$ is a noisy and finite-dimensional approximation of infinite-dimensional data $\Lambda_{\sigma}$, and in most cases it does not actually correspond to any conductivity (as $\Lambda_{\sigma}^{\delta}$ is not in the range of the forward map $\sigma \mapsto \Lambda_{\sigma}$ ). Therefore, EIT reconstruction methods need to be regularized to yield noise-robust results. Regularization is based on complementing the insufficient measurement data by a priori information about the conductivity. 

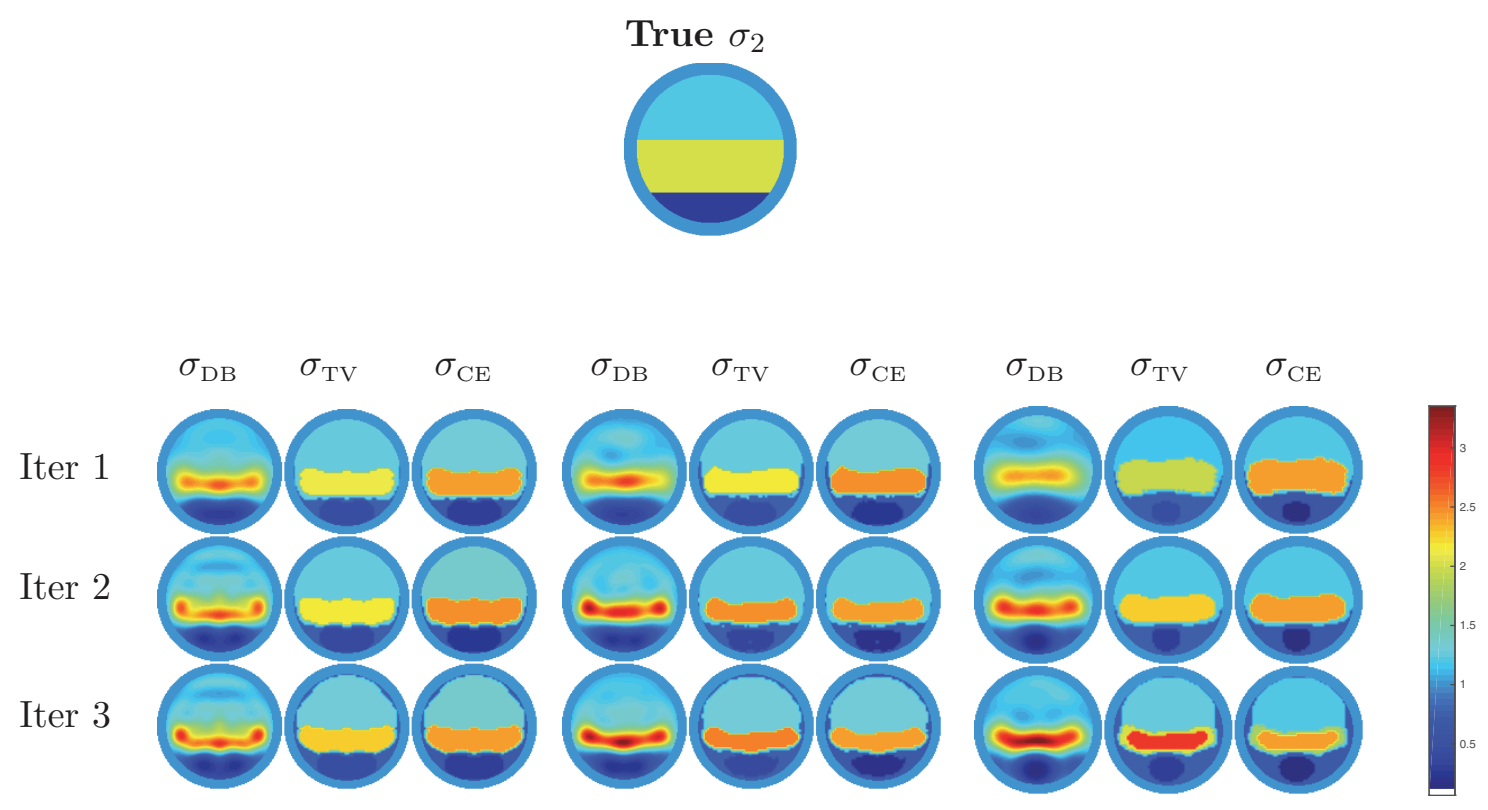

a) no added noise

b) $0.1 \%$ noise

c) $0.75 \%$ noise

Figure 8. This figure shows the real parts of the numerical approximations to the conductivity obtained for the industrial pipe phantom of Example 2, i.e., $\sigma_{2}$. The picture consists of three parts (a)-(c) corresponding to our three cases of added simulated noise in the EIT voltage data: zero added noise, noise of relative amplitude $0.1 \%$, and noise of relative amplitude $0.75 \%$, respectively. For the sake of comparison, the true conductivity $\sigma_{2}$ is displayed above the reconstructions (all on the same color scale). For each noise level, the D-bar reconstruction $\sigma_{D B}^{(j)}$ (left columns), the TV sharpened image $\sigma_{T V}^{(j)}$ (middle columns), and the contrast adjusted TV sharpened images $\sigma_{C E}^{(j)}$ are shown. The first, second, and third rows correspond to the first, second, and third iterations, respectively.

Table 6

The $L^{2}$ relative errors as well as the SSIM values for the zero added noise case for Example 2 with the pipeline phantom $\sigma_{2}$.

$L^{2}$ Relative Error

\begin{tabular}{l|lll}
\hline$j$ & $\sigma_{\mathrm{DB}}^{(j)}$ & $\sigma_{\mathrm{TV}}^{(j)}$ & $\sigma_{\mathrm{CE}}^{(j)}$ \\
\hline 1 & 0.1926 & 0.1926 & 0.2157 \\
2 & 0.1813 & 0.1985 & 0.2286 \\
3 & 0.1862 & 0.2069 & 0.2228 \\
\hline
\end{tabular}

SSIM

\begin{tabular}{l|lll}
\hline$j$ & $\sigma_{\mathrm{DB}}^{(j)}$ & $\sigma_{\mathrm{TV}}^{(j)}$ & $\sigma_{\mathrm{CE}}^{(j)}$ \\
\hline 1 & 0.7097 & 0.7097 & 0.7130 \\
2 & 0.7127 & 0.6976 & 0.6972 \\
3 & 0.7054 & 0.6130 & 0.6128 \\
\hline
\end{tabular}

Currently there are not many regularized reconstruction methods for EIT. The theory of Tikhonov regularization and related variational methods applies to a wide class of nonlinear forward maps [35] but, alas, not to the extremely nonlinear case of EIT. For partial results, see [39, 33, 32]. The enclosure method for detecting convex hulls of inclusions admits a regularization analysis [30], but it only yields partial information (e.g., information about the locations of inclusions rather than their conductivity values). The regularized D-bar 


\section{Table 7}

The $L^{2}$ relative errors as well as the SSIM values for the $0.1 \%$ added noise case for Example 2 with the pipeline phantom $\sigma_{2}$.

$L^{2}$ Relative Error

\begin{tabular}{l|lll}
\hline$j$ & $\sigma_{\mathrm{DB}}^{(j)}$ & $\sigma_{\mathrm{TV}}^{(j)}$ & $\sigma_{\mathrm{CE}}^{(j)}$ \\
\hline 1 & 0.1970 & 0.2176 & 0.2479 \\
2 & 0.2279 & 0.2309 & 0.2305 \\
3 & 0.2329 & 0.2404 & 0.2392 \\
\hline
\end{tabular}

SSIM

\begin{tabular}{l|lll}
\hline$j$ & $\sigma_{\mathrm{DB}}^{(j)}$ & $\sigma_{\mathrm{TV}}^{(j)}$ & $\sigma_{\mathrm{CE}}^{(j)}$ \\
\hline 1 & 0.7292 & 0.6834 & 0.6829 \\
2 & 0.6982 & 0.6826 & 0.6837 \\
3 & 0.6997 & 0.5744 & 0.5653 \\
\hline
\end{tabular}

Table 8

The $L^{2}$ relative errors as well as the SSIM values for the $0.75 \%$ added noise case for Example 2 with the pipeline phantom $\sigma_{2}$.

$L^{2}$ Relative Error

\begin{tabular}{l|lll}
\hline$j$ & $\sigma_{\mathrm{DB}}^{(j)}$ & $\sigma_{\mathrm{TV}}^{(j)}$ & $\sigma_{\mathrm{CE}}^{(j)}$ \\
\hline 1 & 0.2043 & 0.2181 & 0.2617 \\
2 & 0.2255 & 0.2273 & 0.2434 \\
3 & 0.2604 & 0.2758 & 0.2622 \\
\hline
\end{tabular}

SSIM

\begin{tabular}{l|lll}
\hline$j$ & $\sigma_{\mathrm{DB}}^{(j)}$ & $\sigma_{\mathrm{TV}}^{(j)}$ & $\sigma_{\mathrm{CE}}^{(j)}$ \\
\hline 1 & 0.7123 & 0.6908 & 0.6958 \\
2 & 0.6873 & 0.6972 & 0.6960 \\
3 & 0.6933 & 0.5868 & 0.5690 \\
\hline
\end{tabular}

method [37] is the only reconstruction method for which convergence is proven that produces actual conductivity images, but the reconstructions are always smooth because of a nonlinear low-pass filter involved. Indeed, the assumptions of the regularized D-bar method include continuous differentiability of the conductivity, and thus the smoothing is not unexpected.

In many applications of EIT, such as nondestructive testing, the conductivity distribution can be assumed to be piecewise constant. This is approximately the case in medical imaging as well. Therefore, it is desirable to design a regularized reconstruction method producing piecewise constant images.

In this paper, a noise-robust EIT reconstruction method that always results in a piecewise constant image was both presented and tested on simulated noisy EIT data. Therefore, this paper demonstrates that one can achieve the above goal, at least partially. The authors note that this is an initial feasibility study only and do not prove that the new combined method itself is a regularization strategy. Does it seem possible to prove it? The shortcut method currently has no regularization analysis available, but the numerical evidence presented in [5] suggests that the (nonlinearly) low-pass filtered piecewise constant conductivities seem to converge as expected. If that is proven to be the case, perhaps the analytical properties of the TV segmentation could be added to provide an analysis for the method developed here. However, such an analysis is outside the scope of this paper.

Although the scattering data in the extended annuli for $\tau^{(1)}$ and $\tau^{(2)}$ in each example is not identical to that of $\tau_{\mathrm{B}}$, nonetheless, the subsequent corresponding conductivity reconstructions show marked improvements in the locations, sharpness of edges, and conductivity values of the inclusions. Take particular note of the $0.75 \%$ noise case of Example 1 where in iterations 1 and 2 the images $\sigma_{\mathrm{DB}}, \sigma_{\mathrm{TV}}$, and $\sigma_{\mathrm{CE}}$ all contain a strong artifact above the heart which 
is not present after an additional iteration (i.e., in iteration 3). In Example 2, we see that after a single sharpening of the original D-bar image the corresponding $\sigma_{\mathrm{TV}}^{(1)}$ is enough for the goal of distinguishing between oil, water, and sand in the pipeline. These two cases (shown for various levels of noise) demonstrate the flexibility of the algorithm: for some cases it is appropriate to be applied iteratively for improvements and to remove artifacts, whereas in other cases a single sharpening iteration is adequate.

Additional modifications to the proposed method can be easily applied. In particular, in lieu of fixing the maximal number of iterations $J_{\text {iter }}$ beforehand, alternative stopping criteria could be applied. For example, for $j>1$, instead of asking above whether $j=J_{\text {iter }}$, return $\sigma_{\mathrm{CE}}^{(j)}$ as the final image if

$$
\left\|\sigma_{\mathrm{CE}}^{(j)}-\sigma_{\mathrm{CE}}^{(j-1)}\right\|_{l^{2}} /\left\|\sigma_{\mathrm{CE}}^{(j)}\right\|_{l^{2}}<\text { thresh }
$$

for some specified threshold thresh. Another option concerns the radii $R$ and $\widetilde{R}$. In the examples presented here, the radii $R$ and $\widetilde{R}$ are fixed throughout the algorithm for each data set $\Lambda_{\sigma}$. An alternative approach could compute the Beltrami scattering data on progressively larger annuli so that in step (e) of the algorithm in Figure 3, the new scattering data $\widetilde{\tau}^{(j)}(k)$ is computed for $R-1<|k| \leq \widetilde{R}+(j-1) \Delta R$ given a fixed stepsize $\Delta R>0(j \geq 1)$. Such approaches, while interesting, are outside the scope of this work.

\section{REFERENCES}

[1] K. Astala, J.L. Mueller, L. Päivärinta, A. Perämäki, and S. Siltanen, Direct electrical impedance tomography for nonsmooth conductivities, Inverse Probl. Imaging, 5 (2011), pp. 531-549, http://dx. doi.org/10.3934/ipi.2011.5.531.

[2] K. Astala, J.L. Mueller, L. Päivärinta, And S. Siltanen, Numerical computation of complex geometrical optics solutions to the conductivity equation, Appl. Comput. Harmon. Anal., 29 (2010), pp. 391-403, http://dx.doi.org/10.1016/j.acha.2009.08.001.

[3] K. Astala And L. P̈̈IVÄRInta, A boundary integral equation for Calderón's inverse conductivity problem, Collect. Math., 57 (2006), pp. 127-139.

[4] K. Astala And L. PÄIvÄrinta, Calderón's inverse conductivity problem in the plane, Ann. of Math. (2), 163 (2006), pp. 265-299, http://dx.doi.org/10.4007/annals.2006.163.265.

[5] K. Astala, L. PÄivärinta, J.M. Reyes, and S. Siltanen, Nonlinear Fourier analysis for discontinuous conductivities: Computational results, J. Comput. Phys., 276 (2014), pp. 74-91, http://dx.doi.org/ 10.1016/J.JCP.2014.07.032.

[6] G. Aubert And P. Kornprobst, Mathematical Problems in Image Processing: Partial Differential Equations and the Calculus of Variations, Appl. Math. Sci. 147, Springer, New York, 2006.

[7] E. BAE, J. YUAN, AND X.C. TAI, Global minimization for continuous multiphase partitioning problems using a dual approach, Int. J. Comput. Vis., 92 (2011), pp. 112-129, http://dx.doi.org/10.1007/ s11263-010-0406-y.

[8] L. Bar, T.F. Chan, G. Chung, M. Jung, N. Kiryati, R. Mohieddine, N. Sochen, And L.A. VESE, Mumford and Shah model and its applications to image segmentation and image restoration, in Handbook of Mathematical Methods in Imaging, Springer, New York, 2011, pp. 1095-1157, http:// dx.doi.org/10.1007/978-1-4939-0790-8_25.

[9] X. Bresson, S. Esedoglu, P. Vandergheynst, J.P. Thiran, and S. Osher, Fast global minimization of the active contour/snake model, J. Math. Imaging Vision, 28 (2007), pp. 151-167, http://dx.doi. org/10.1007/s10851-007-0002-0. 
[10] A.-P. Calderón, On an inverse boundary value problem, in Seminar on Numerical Analysis and Its Applications to Continuum Physics (Rio de Janeiro, 1980), Sociedade Brasileira de Matemática, Rio de Janeiro, 1980, pp. 65-73.

[11] A. Chambolle, S.E. Levine, And B.J. Lucier, An upwind finite-difference method for total variationbased image smoothing, SIAM J. Imaging Sci., 4 (2011), pp. 277-299, http://dx.doi.org/10.1137/ 090752754 .

[12] A. Chambolle And T. Pock, A first-order primal-dual algorithm for convex problems with applications to imaging, J. Math. Imaging Vision, 40 (2011), pp. 120-145, http://dx.doi.org/10.1007/ s10851-010-0251-1.

[13] T.F. Chan And S. Esedō̄LU, Aspects of total variation regularized $L^{1}$ function approximation, SIAM J. Appl. Math., 65 (2005), pp. 1817-1837, http://dx.doi.org/10.1137/040604297.

[14] T.F. Chan, S. Esedō̄Lu, And M. Nikolova, Algorithms for finding global minimizers of image segmentation and denoising models, SIAM J. Appl. Math., 66 (2006), pp. 1632-1648, http://dx.doi.org/ $10.1137 / 040615286$.

[15] T.F. Chan And J. Shen, Image Processing and Analysis: Variational, PDE, Wavelet, and Stochastic Methods, SIAM, Philadelphia, 2005, http://dx.doi.org/10.1137/1.9780898717877.

[16] T.F. CHAN AND X.-C. TAI, Level set and total variation regularization for elliptic inverse problems with discontinuous coefficients, J. Comput. Phys., 193 (2004), pp. 40-66, http://dx.doi.org/10.1016/j.jcp. 2003.08.003.

[17] T.F. Chan And L.A. Vese, Active contours without edges, IEEE Trans. Image Process., 10 (2001), pp. 266-277, http://dx.doi.org/10.1109/83.902291.

[18] M. Cheney, D. Isaacson, and J.C. Newell, Electrical impedance tomography, SIAM Rev., 41 (1999), pp. 85-101, http://dx.doi.org/10.1137/S0036144598333613.

[19] E.T. Chung, T.F. Chan, And X.-C. TAI, Electrical impedance tomography using level set representation and total variational regularization, J. Comput. Phys., 205 (2005), pp. 357-372, http://dx.doi.org/ 10.1016/j.jcp.2004.11.022.

[20] M. DeAngelo And J.L. Mueller, $2 d$ D-bar reconstructions of human chest and tank data using an improved approximation to the scattering transform, Physiol. Meas., 31 (2010), pp. 221-232, http:// dx.doi.org/10.1088/0967-3334/31/2/008.

[21] D.C. Dobson And F. Santosa, An image enhancement technique for electrical impedance tomography, Inverse Problems, 10 (1994), pp. 317-334, http://dx.doi.org/10.1088/0266-5611/10/2/008.

[22] M. Dodd And J.L. Mueller, A real-time d-bar algorithm for 2-d electrical impedance tomography data, Inverse Probl. Imaging, 8 (2014), pp. 1013-1031, http://dx.doi.org/10.3934/ipi.2014.8.1013.

[23] E. Esser, X. Zhang, AND T.F. ChAn, A general framework for a class of first order primal-dual algorithms for convex optimization in imaging science, SIAM J. Imaging Sci., 3 (2010), pp. 10151046, http://dx.doi.org/10.1137/09076934X.

[24] L.D. FADDEEv, Increasing solutions of the Schrödinger equation, Soviet Phys. Dokl., 10 (1966), pp. 10331035.

[25] S.J. Hamilton, A. Hauptmann, and S. Siltanen, A data-driven edge-preserving D-bar method for electrical impedance tomography, Inverse Probl. Imaging, 8 (2014), pp. 1053-1072, http://dx.doi.org/ 10.3934/ipi.2014.8.1053.

[26] S.J. Hamilton, M. Lassas, and S. Siltanen, A direct reconstruction method for anisotropic electrical impedance tomography, Inverse Problems, 30 (2014), 075007, http://dx.doi.org/10.1088/0266-5611/ $30 / 7 / 075007$.

[27] B. Harrach And M. Ullrich, Monotonicity-based shape reconstruction in electrical impedance tomography, SIAM J. Math. Anal., 45 (2013), pp. 3382-3403, http://dx.doi.org/10.1137/120886984.

[28] C.N.L. Herrera, M.F.M. Vallejo, J.L. Mueller, And R.G. Lima, Direct 2-d reconstructions of conductivity and permittivity from EIT data on a human chest, IEEE Trans. Med. Imag., 34 (2015), pp. 267-274, http://dx.doi.org/10.1109/TMI.2014.2354333.

[29] M. Huhtanen And A. Perämäki, Numerical solution of the R-linear Beltrami equation, Math. Comp., 81 (2012), pp. 387-397, https://www.researchgate.net/publication/220576569_Numerical_solution_of_ the_R-linear_Beltrami_equation.

[30] M. Ikehata And S. Siltanen, Electrical impedance tomography and Mittag-Leffler's function, Inverse Problems, 20 (2004), pp. 1325-1348, http://dx.doi.org/10.1088/0266-5611/20/4/019. 
[31] D. Isaacson, J.L. Mueller, J.C. Newell, and S. Siltanen, Imaging cardiac activity by the D-bar method for electrical impedance tomography, Physiol. Meas., 27 (2006), pp. S43-S50, http://dx.doi. org/10.1088/0967-3334/27/5/S04.

[32] B. Jin AND P. MAASs, An analysis of electrical impedance tomography with applications to Tikhonov regularization, ESAIM Control Optim. Calc. Var., 18 (2012), pp. 1027-1048, http://dx.doi.org/10. 1051/cocv/2011193.

[33] B. JIN AND P. MAAss, Sparsity regularization for parameter identification problems, Inverse Problems, 28 (2012), 123001, http://dx.doi.org/10.1088/0266-5611/28/12/123001.

[34] J.P. Kaipio, V. Kolehmainen, E. Somersal, And M. Vauhkonen, Statistical inversion and Monte Carlo sampling methods in electrical impedance tomography, Inverse Problems, 16 (2000), pp. 14871522, http://dx.doi.org/10.1088/0266-5611/16/5/321.

[35] B. Kaltenbacher, A. Neubauer, And O. Scherzer, Iterative Regularization Methods for Nonlinear Ill-Posed Problems, Radon Ser. Comput. Appl. Math. 6, De Gruyter, Berlin, 2008.

[36] K. Knudsen, M. Lassas, J.L. Mueller, And S. Siltanen, D-bar method for electrical impedance tomography with discontinuous conductivities, SIAM J. Appl. Math., 67 (2007), pp. 893-913, http:// dx.doi.org/10.1137/060656930.

[37] K. Knudsen, M. Lassas, J.L. Mueller, and S. Siltanen, Regularized D-bar method for the inverse conductivity problem, Inverse Probl. Imaging, 3 (2009), pp. 599-624, http://dx.doi.org/10.3934/ipi. 2009.3.599.

[38] K. Knudsen, J.L. Mueller, and S. Siltanen, Numerical solution method for the dbar-equation in the plane, J. Comput. Phys., 198 (2004), pp. 500-517, http://dx.doi.org/10.1016/j.jcp.2004.01.028.

[39] A. Lechleiter AND A. Rieder, Newton regularizations for impedance tomography: Convergence by local injectivity, Inverse Problems, 24 (2008), 065009, http://dx.doi.org/10.1088/0266-5611/24/6/065009.

[40] J.L. Mueller And S. Siltanen, Direct reconstructions of conductivities from boundary measurements, SIAM J. Sci. Comput., 24 (2003), pp. 1232-1266, http://dx.doi.org/10.1137/S1064827501394568.

[41] J.L. Mueller And S. Siltanen, Linear and Nonlinear Inverse Problems with Practical Applications, SIAM, Philadelphia, 2012, http://dx.doi.org/10.1137/1.9781611972344.

[42] D. Mumford And J. Shah, Optimal approximations by piecewise smooth functions and associated variational problems, Comm. Pure Appl. Math., 42 (1989), pp. 577-685, http://dx.doi.org/10.1002/cpa. 3160420503.

[43] E.K. Murphy, J.L. Mueller, And J.C. Newell, Reconstructions of conductive and insulating targets using the D-bar method on an elliptical domain, Physiol. Meas., 28 (2007), pp. S101-S144, http://dx. doi.org/10.1088/0967-3334/28/7/S08.

[44] A.I. NaChman, Global uniqueness for a two-dimensional inverse boundary value problem, Ann. of Math. (2), 143 (1996), pp. 71-96, http://dx.doi.org/10.2307/2118653.

[45] C.D. Perttunen, D.R. Jones, And B.E. Stuckman, Lipschitzian optimization without the Lipschitz constant, J. Optim. Theory Appl., 79 (1993), pp. 157-181, http://dx.doi.org/10.1007/BF00941892.

[46] T. Pock, A. Chambolle, D. Cremers, and H. Bischof, A convex relaxation approach for computing minimal partitions, in Proceedings of the IEEE Conference on Computer Vision and Pattern Recognition, 2009, pp. 810-817, http://dx.doi.org/10.1109/CVPR.2009.5206604.

[47] L. Rondi And F. SAntosa, Enhanced electrical impedance tomography via the Mumford-Shah functional, ESAIM Control Optim. Calc. Var., 6 (2001), pp. 517-538, http://dx.doi.org/10.1051/cocv:2001121.

[48] L.I. Rudin, S. Osher, And E. FAtemi, Nonlinear total variation based noise removal algorithms, Phys. D, 60 (1992), pp. 259-268, http://dx.doi.org/10.1016/0167-2789(92)90242-F.

[49] B. Sandberg And T.F. Chan, A logic framework for active contours on multi-channel images, J. Vis. Commun. Image Represent., 16 (2005), pp. 333-358, http://dx.doi.org/10.1016/j.jvcir.2004.08.005.

[50] S. Siltanen, J. Mueller, And D. IsaAcson, An implementation of the reconstruction algorithm of A. Nachman for the 2-D inverse conductivity problem, Inverse Problems, 16 (2000), pp. 681-699, http://dx.doi.org/10.1088/0266-5611/17/5/501.

[51] J. Sylvester and G. Uhlmann, A global uniqueness theorem for an inverse boundary value problem, Ann. of Math. (2), 125 (1987), pp. 153-169, http://dx.doi.org/10.2307/1971291.

[52] C. TAI, X. Zhang, And Z. Shen, Wavelet frame based multiphase image segmentation, SIAM J. Imaging Sci., 6 (2013), pp. 2521-2546, http://dx.doi.org/10.1137/120901751.

[53] N.M. TAnushev And L.A. Vese, A piecewise-constant binary model for electrical impedance tomography, 
Inverse Probl. Imaging, 1 (2007), pp. 423-435, http://dx.doi.org/10.3934/ipi.2007.1.423.

[54] K. VAN DEN DOel AND U.M. Ascher, On level set regularization for highly ill-posed distributed parameter estimation problems, J. Comput. Phys., 216 (2006), pp. 707-723, http://dx.doi.org/10.1016/j.jcp. 2006.01.022.

[55] Z. Zhou, G. Sato dos Santos, T. Dowrick, J. Avery, Z. Sun, H. Xu, And D.S. Holder, Comparison of total variation algorithms for electrical impedance tomography, Physiol. Meas., 36 (2015), pp. 1193-1209, http://dx.doi.org/10.1088/0967-3334/36/6/1193. 